\title{
The infrared spectrograph on the Spitzer Space Telescope
}

\section{James R. Houck, Thomas L. Roellig, Jeff Van Cleve, William J. Forrest, Terry L. Herter, et al.}

James R. Houck, Thomas L. Roellig, Jeff Van Cleve, William J. Forrest, Terry L. Herter, Charles R. Lawrence, Keith Matthews, Harold J. Reitsema, B. Thomas Soifer, Dan M. Watson, Dan Weedman, Marty Huisjen, John R. Troeltzsch, Donald J. Barry, J. Bernard-Salas, Craig Blacken, Bernhard Rainer Brandl, Vassilis Charmandaris, Daniel Devost, George E. Gull, Peter Hall, Charles P. Henderson, S. James U. Higdon, Bruce E. Pirger, Justin Schoenwald, Greg C. Sloan, Keven I. Uchida, Philip N. Appleton, Lee Armus, Martin J. Burgdorf, Sergio B. Fajardo-Acosta, Carl J. Grillmair, Jim G. Ingalls, Patrick W. Morris, Harry I. Teplitz, "The infrared spectrograph on the Spitzer Space Telescope," Proc. SPIE 5487, Optical, Infrared, and Millimeter Space Telescopes, (12 October 2004); doi: 10.1117/12.550517

Event: SPIE Astronomical Telescopes + Instrumentation, 2004, Glasgow, United Kingdom 


\title{
The Infrared Spectrograph on the Spitzer Space Telescope*
}

\author{
J. R. Houck ${ }^{\mathrm{a}}$, T. L. Roellig ${ }^{\mathrm{b}}$, J. van Cleve ${ }^{\mathrm{c}}$, W. J. Forrest ${ }^{\mathrm{d}}$, T. Herter ${ }^{\mathrm{a}}$, C. R. Lawrence ${ }^{\mathrm{e}}$, K. Matthews ${ }^{\mathrm{f}}$, \\ H. J. Reitsema ${ }^{\mathrm{c}}$, B. T. Soifer ${ }^{\mathrm{g}}$, D. M. Watson ${ }^{\mathrm{d}}$, D. Weedman ${ }^{\mathrm{a}}$, M. Huisjen ${ }^{\mathrm{c}}$, J. Troeltzsch ${ }^{\mathrm{c}}$, \\ D. J. Barry ${ }^{\mathrm{a}}$, J. Bernard-Salas ${ }^{\mathrm{a}}$, C. E. Blacken ${ }^{\mathrm{a}}$, B. R. Brandl ${ }^{\mathrm{h}}$, V. Charmandaris ${ }^{\mathrm{a}, \mathrm{i}}$, D. Devost ${ }^{\mathrm{a}}$, \\ G. E. Gull ${ }^{\mathrm{a}}$, P. Hall ${ }^{\mathrm{a}}$, C. P. Henderson ${ }^{\mathrm{a}}$, S. J. U. Higdon ${ }^{\mathrm{a}}$, B. E. Pirger ${ }^{\mathrm{a}}$, J. Schoenwald ${ }^{\mathrm{a}}$, G. C. Sloan ${ }^{\mathrm{a}}$, \\ K. I. Uchida ${ }^{\mathrm{a}}$, P. N. Appleton ${ }^{\mathrm{g}}$, L. Armus ${ }^{\mathrm{g}}$, M. J. Burgdorf ${ }^{\mathrm{g}}$, S. B. Fajardo-Acosta ${ }^{\mathrm{g}}$, C. J. Grillmair ${ }^{\mathrm{g}}$, \\ J. G. Ingalls ${ }^{\mathrm{g}}$, P. W. Morris ${ }^{\mathrm{g} . \mathrm{j}}$, H. I. Teplitz ${ }^{\mathrm{g}}$
}

a Cornell University, Astronomy Department, Ithaca, NY 14853-6801, jrh13@cornell.edu

b NASA Ames Research Center, MS 245-6, Moffett Field, CA 94035-1000

c Ball Aerospace \& Technologies Corp., 1600 Commerce St., Boulder, CO 80301

d University of Rochester, Department of Physics \& Astronomy, Rochester, NY 14627

e Caltech, Jet Propulsion Laboratory, Mailcode 169-327, Pasadena, CA 91125

f Caltech, Palomar Observatory, Pasadena, CA 91125

g Caltech, Spitzer Science Center, MS 220-6, Pasadena, CA 91125

h Leiden University, 2300 RA Leiden, The Netherlands

i Chercheur Associ'e, Observatoire de Paris, F-75014 Paris, France

j NASA Herschel Science Center, IPAC/Caltech, MS 100-22, Pasadena, CA 91125

*The IRS was a collaborative venture between Cornell University and Ball Aerospace Corporation funded by NASA through the Jet Propulsion Laboratory and the Ames Research Center.

\begin{abstract}
The Infrared Spectrograph (IRS) is one of three science instruments on the Spitzer Space Telescope. The IRS comprises four separate spectrograph modules covering the wavelength range from 5.3 to $38 \mu \mathrm{m}$ with spectral resolutions, $R \sim 90$ and 650, and it was optimized to take full advantage of the very low background in the space environment. The IRS is performing at or better than the pre-launch predictions. An autonomous target acquisition capability enables the IRS to locate the mid-infrared centroid of a source, providing the information so that the spacecraft can accurately offset that centroid to a selected slit. This feature is particularly useful when taking spectra of sources with poorly known coordinates. An automated data reduction pipeline has been developed at the Spitzer Science Center.
\end{abstract}

Subject headings: Instrumentation: spectrographs - Infrared: general

\section{INTRODUCTION}

The Infrared Spectrograph (IRS) on the Spitzer Space Telescope provides capabilities for low and moderate-resolution spectroscopy, as well as some limited imaging capabilities. The primary objective in the design of the IRS was to take maximal advantage of the very low background conditions provided by the space environment. The mid-infrared background in the Spitzer orbit is approximately $10^{6}$ times smaller than the background at a modern ground-based facility. If the benefit of this reduced background is fully achieved, the enhancement in sensitivity is $\sim 10^{3}$, while the speed enhancement is $\sim 10^{6}$. The low-resolution modules described below very nearly achieve the full benefit of the space environment, while the high-resolution modules are detector noise limited.

The infrared spectrograph for Spitzer was heavily cost constrained. To reduce the number of initial design studies, a set of axioms was developed ${ }^{1}$. These axioms were: 
1. Spitzer is a cost driven mission.

2. Boeing Si:As and Si:Sb BIB arrays will be used.

3. The IRS will have aluminum structure and optics.

4. Only simple optics will be used.

- Only surfaces of revolution (may be off axis)

- $\quad$ Flat gratings

5. There will be no moving parts and no adjustments.

6. The IRS will be redundant only for credible single point failures.

7. The design will strive for an observing efficiency of $>80 \%$.

8. The IRS will be capable of internal health assessment.

The resulting design consists of four separate modules whose basic characteristics are summarized in Table 1 . The finished, tested, and qualified modules are shown mounted on their flight baseplate in Figure $1^{2}$. A sample peak-up image and the spectrum of the blue compact galaxy, SBS 0335-052 from the short low and long low modules are shown in Figure 2.

The low-resolution modules are long slit spectrographs with two slits each. Light passing through one slit is dispersed in first order while light passing through the other slit is dispersed in first and second order. Because the latter slit includes a small portion of the first order light, there is significant overlap between spectra taken through the two slits. This helps establish the relative amplitudes of the first and second order spectra. The high-resolution modules have short slits that are roughly twice as long as their widths. A first order grating is used as the predisperser for the echelle that operates in $11^{\text {th }}$ through $20^{\text {th }}$ orders $^{3 \& 4}$. The Short-Low module also has two imaging apertures to aid in source acquisition and telescope pointing. The interference filters used to block overlapping orders and to define the imaging band passes were manufactured by Optical Coating Laboratories, OCLI. The physical locations and orientations of the slits and apertures in the Spitzer focal plane can be found in the Spitzer Observer's Manual (SOM) ${ }^{5}$.

Table 1. Module Characteristics

\begin{tabular}{|l|c|c|c|c|}
\hline \multicolumn{1}{|c|}{ Module } & $\begin{array}{c}\text { Wavelength Range } \\
(\boldsymbol{\mu m})\end{array}$ & $\begin{array}{c}\text { Pixel Size } \\
(\mathbf{a r c s e c})\end{array}$ & $\begin{array}{c}\text { Slit Width x Length } \\
(\mathbf{a r c s e c})\end{array}$ & $\begin{array}{c}\text { Spectral Resolution } \\
(\boldsymbol{\lambda} / \Delta \boldsymbol{\lambda})\end{array}$ \\
\hline Short-Low & $5.2-8.7$ (SL2) & 1.8 & $3.6 \times 57$ & $\sim 80$ to 128 \\
& $7.4-14.5(\mathrm{SL} 1)$ & 1.8 & $3.7 \times 57$ & $\sim 64$ to 128 \\
\hline Blue Peak-Up & $13.5-18.7$ (PU-B) & 1.8 & $56 \times 80$ & $\sim 3$ \\
\hline Red Peak-Up & $18.5-26.0$ (PU-R) & 1.8 & $54 \times 82$ & $\sim 3$ \\
\hline Long -Low & $14.0-21.3$ (LL2) & 5.1 & $10.5 \times 168$ & $\sim 80$ to 128 \\
& $19.5-38.0(\mathrm{LL} 1)$ & 5.1 & $10.7 \times 168$ & $\sim 600$ \\
\hline Short - High & $9.9-19.6$ & 2.3 & $4.7 \times 11.3$ & $\sim 600$ \\
\hline Long - High & $18.7-37.2$ & 4.5 & $11.1 \times 22.3$ & $\sim 600$ \\
\hline
\end{tabular}

\section{DETECTORS}

The detectors were developed and manufactured by Boeing North America as part of an ongoing collaboration with Cornell University. Their general characteristics are summarized in Table 2.

Table 2. Detector Characteristics

\begin{tabular}{|l|c|c|l|}
\hline \multicolumn{1}{|c|}{ Parameter } & Si:As & Si:Sb & \multicolumn{1}{c|}{ Units } \\
\hline Format & $128 \times 128$ & $128 \times 128$ & Pixels \\
\hline Pixel Size & $75 \times 75$ & $75 \times 75$ & $\mu \mathrm{m}$ \\
\hline Read noise & 30 & 30 & $\mathrm{e}^{-} / \mathrm{read}$ \\
\hline Dark-Current & 10 & 30 & $\mathrm{e}^{-} / \mathrm{sec}$ \\
\hline DQE & 50 & 40 & $\%$ \\
\hline
\end{tabular}




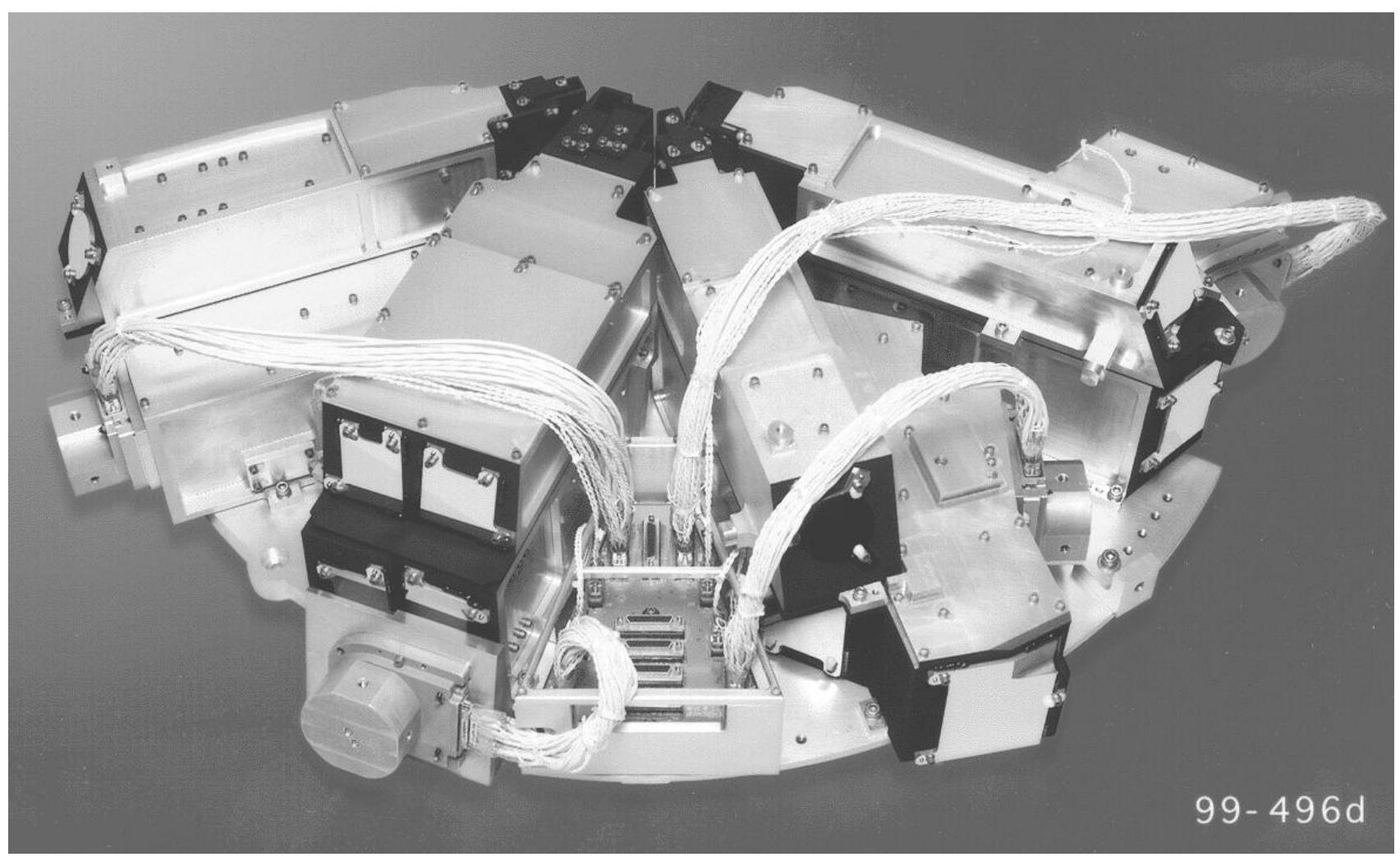

Figure 1. The four IRS modules mounted on their flight baseplate.
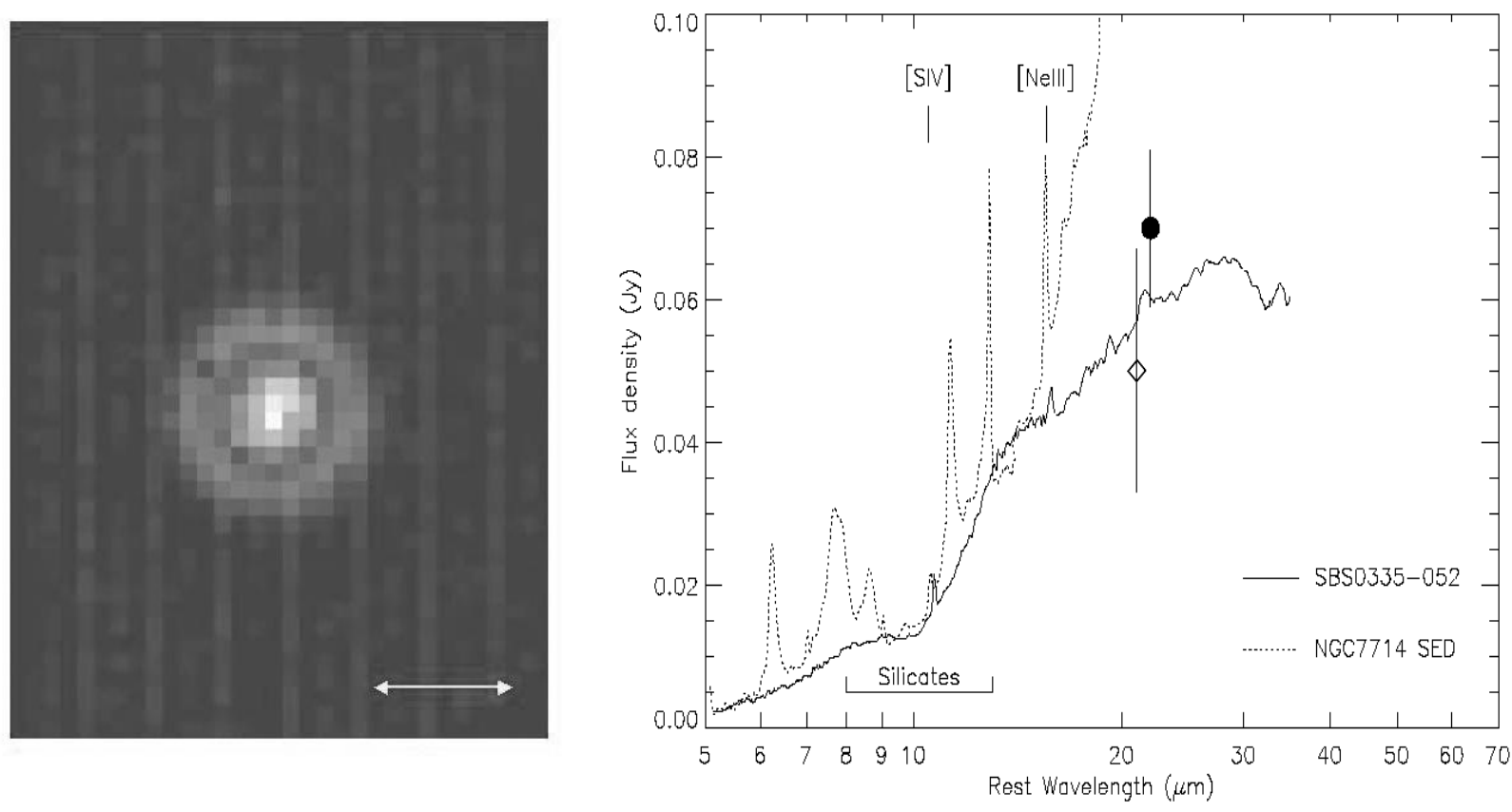

Figure 2. The left hand image is the peak-up image for the blue compact galaxy SBS 0335-052. The double ended arrow indicates approximately $30 \operatorname{arcsec}(7.5 \mathrm{kpc})$. The combined short low and long low spectra are shown in the right hand panel. The solid circle is the photometry from the red peak-up image. The diamond is from Gemini. 
When the IRS is operating, all four of its detector arrays are clocked simultaneously, but it is only possible to capture data from one array at a time. Two techniques are used in data collection; the double correlated sampling (DCS mode) and raw data collection (Raw mode, or "sample up the ramp"). Science data are collected in Raw mode while Peak-Up employs DCS. In DCS mode following an initial series of bias boost and reset frames, each pixel is sampled, and then after a number of non-destructive spins through the array each pixel is sampled again via a destructive read and the difference between the two samples is stored as an 128x128 pixel image. In the RAW mode after the same initial bias boost, reset frames and first sampling of pixels there are a number of spin frames followed by a non-destructive read. This pattern of spins and reads is repeated $n$ times. When all pixels are sampled again the result is a $128 \times 128 \times n$ cube, where $n$ is the number of non-destructive reads, and the values are $n=4,8$, or 16 . A final $128 \times 128$ image is created by calculating the signal slope of each pixel of the Raw data cube.

A more complete discussion of the performance and clocking modes of the detectors can be found in the $\mathrm{SOM}^{5}$ and Van Cleve et al. ${ }^{6}$

\section{FABRICATION}

The individual modules are each about the size of a shoebox. The housings were manufactured in two parts: an upper and lower housing. The housings were hogged out of aluminum blocks all cut from the same plate. They were first rough machined, and then heat treated to remove the stresses produced by the rolling and machining processes. The heat-treated parts were then finish machined. Each module has between 8 and 12 baffle plates mounted more or less perpendicular to the ray bundles. Each baffle has 1 to 4 holes to pass the bundles at different locations along the optical path. The interiors of the housings and their baffles were painted black. The upper and lower housings are held together in a semi-kinematic way with screws and pins. A labyrinth joint between the housing sections allows trapped air to escape during pump down while preventing photons from entering the housings. The mirrors and gratings are mounted from the outside permitting easy replacement of an optic if need be. Three diamond turned tabs on the optics are held to matching lapped bosses on the housing exteriors with screws and pins. The backs of the optics were also diamond turned, they were checked interferometrically before and after an optic's installation to determine if the optics had been distorted during the installation process. A similar three-pad scheme was used to mount the filter and slit holders to the housings. There are no provisions for adjusting the optics or apertures. A spacer plate between the housing and the focalplane array is custom machined to achieve proper detector focus and centering.

\section{OPTICAL TESTING}

Prior to launch, the IRS was extensively tested for over a year in a series of fourteen separate cool downs in the IRS test facility at Ball Aerospace and Technologies Corp. In each of the approximately one weeklong cool downs, one or more of the IRS modules was tested. Over this period there were hundreds of hours of testing, during which many thousands of images were recorded. The general testing procedures are described below. The requirements and actual in-flight performance figures are discussed below and updated in the $\mathrm{SOM}^{5}$

The ideal test environment would be a fully cooled $(\sim 4 \mathrm{~K})$ test chamber that could accommodate the IRS cryogenic assemblies, and a series of test sources which delivered a properly configured beam to the module entrance apertures with a wide variety of selectable spectral and photometric characteristics. However, the cost of such a facility in terms of complexity, certification, schedule, and budget impact was prohibitive. In fact, just designing, building, and certifying such a cryogenic facility would be a task nearly as difficult as building the IRS itself. In keeping with the IRS axioms, a modular test approach was adopted in which many different optical configurations could be employed to generate a large quantity of data to certify the IRS modules. The turn-around time for any one cool-down was kept to a minimum. The quickest turn-around in the actual testing program was just six days.

The testing of the IRS was performed in the large clean room in the Fisher Test Building at Ball. The basic setup consisted of a Dewar capable of holding the entire IRS cold assembly of four modules, and the cold interface board mounted on the IRS flight baseplate. In addition, there were a number of ancillary infrared sources external to the Dewar that could be used to feed light into the IRS. 


\subsection{Janis Test Dewar}

The workhorse of the IRS test facility was the Janis Dewar. A schematic view of the filter stack inside of the Dewar is shown in Figure 3. The modules were mounted on the IRS baseplate that was in turn mounted on a cold fin inside the Dewar. The IRS was surrounded by a LHe cooled shield which was painted black on the inside. The IRS detectors routinely reached temperatures of $\sim 5.5 \mathrm{~K}$.

Optical access to the modules was through a KRS-5 vacuum window, a set of $\mathrm{LN}_{2}$ and $\mathrm{LHe}$ cooled thin film neutral density filters on silicon substrates, and a light-tight manually-operated LHe-cooled door. Care was taken to minimize reflections between the ND filters by tipping them and providing adequate black surface area to absorb radiation reflected by the filters before it could get back into the optical path. Nonetheless, off-axis $\mathrm{LN}_{2}$ radiation can be reflected into the optical path by the inner side of the $\mathrm{LN}_{2} \mathrm{ND}$ filter. This is a significant signal. However, the images of external sources are generally differences with the source "on" and "off." This subtraction removes the "false" cold radiation signal. Of course, the ND filters are absent in the flight configuration!

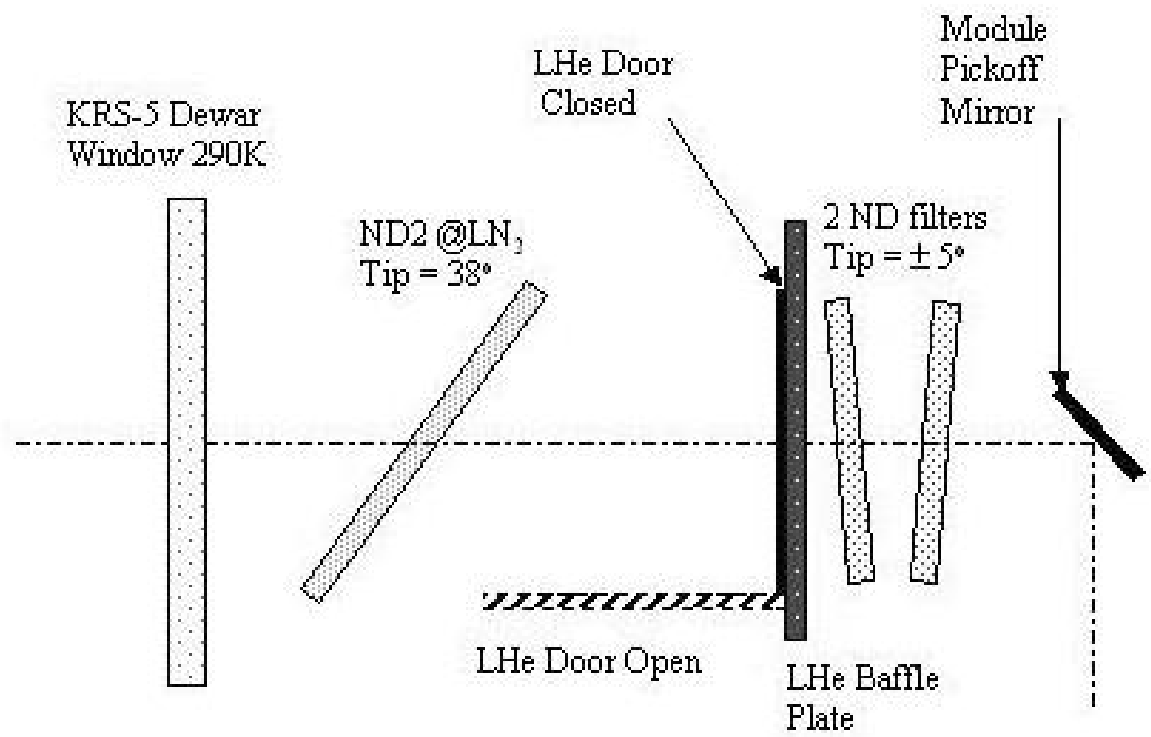

Figure 3. A schematic view of the window and filter stack in the Janis test Dewar is shown. While the $\mathrm{LN}_{2}$ cooled filter was common to all modules, the LHe cooled filters were optimized for each individual modules. 


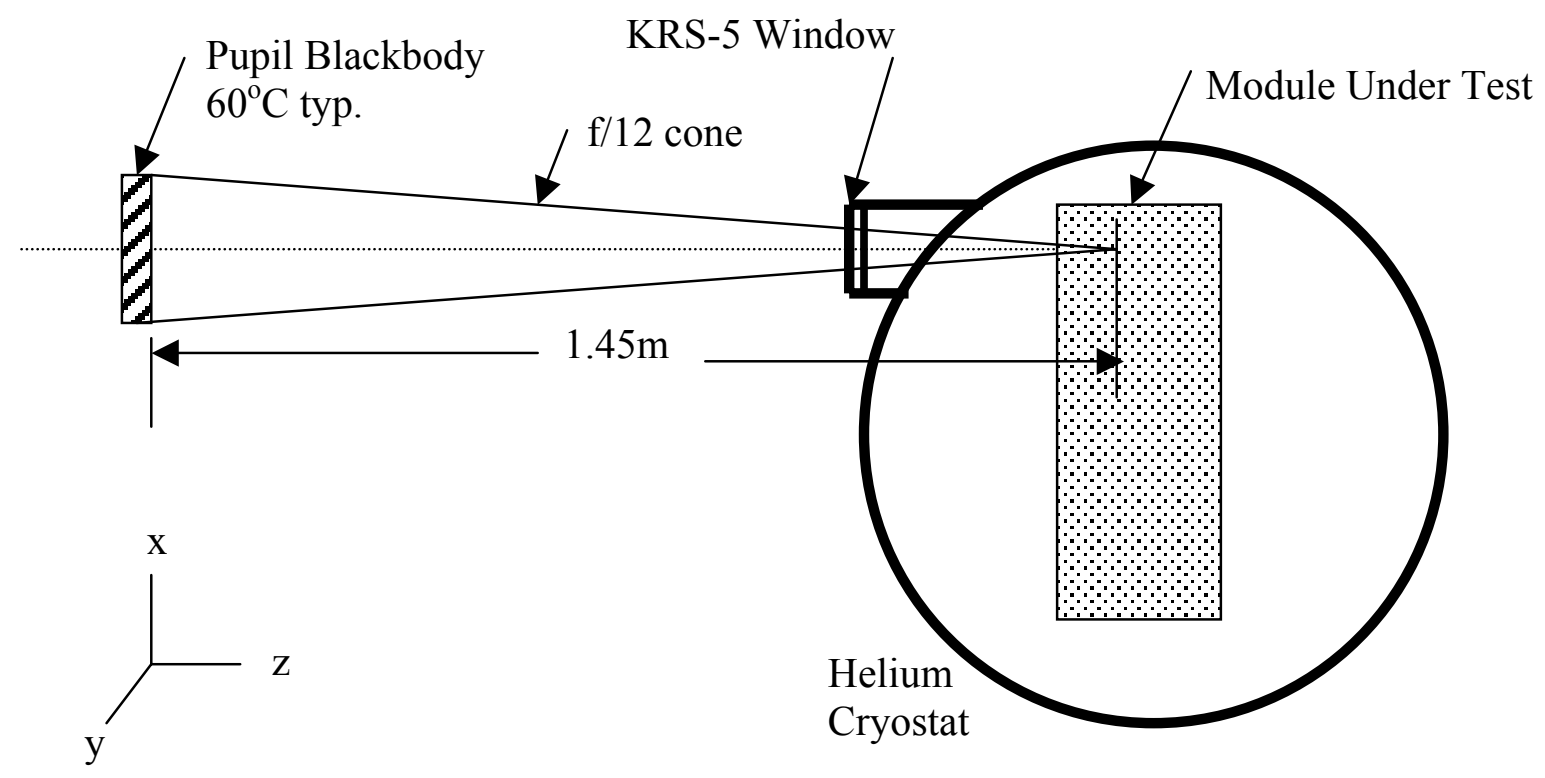

Figure 4. The Pupil Blackbody and the Janis Dewar.

When the LHe door is closed the background in the modules is too small to be measured accurately. In this condition the read-noise and an upper limit to the dark-current performance were measured.

\subsection{Pupil blackbody}

A blackbody source with the diameter and relative location of the Spitzer Cryogenic Telescope Assembly, CTA, exit pupil produced a beam mimicking the radiation coming from an extended source as viewed by the CTA, see Figure 4 . This custom-made pupil blackbody, PBB, could be moved toward and away from its nominal position ( \pm Z) as well as in the plane of the CTA exit pupil $( \pm X, Y)$ to map the shape of the modules' f-beams. This system was used to verify that the modules were all pointed in the same direction and toward the position of the exit pupil. Tests were performed to measure the emissivity of the PBB.

\subsection{Point source projector}

An all-reflecting optical system, using a spherical mirror, projected a point source image into the entrance apertures of the modules. Data from these tests were used to verify the focus of the modules. The point source projector was also used to make an end-to-end test of the peak-up system. A spot was projected into one of the peak-up windows, and the software was commanded to perform a peak-up operation. The source location returned by the peak-up software in the $\mathrm{CE}$ was then compared to the location of the point source image as determined by more conventional image processing. The accuracies of these comparisons were consistent with the thousands of computer simulations of the peak-up process that were performed on simulated peak-up images.

\subsection{Monochromator}

A commercial grating monochromator was available to feed light into the point source projector and from there to the module apertures. The monochromator was run at high order whenever possible to project multiple wavelengths into the apertures at once. These tests determined the dispersion curves for the spectrographs. A variety of slits and gratings were available for the monochromator to cover the IRS' wavelengths and resolutions. In addition, a set of $\lambda / \Delta \lambda \sim 10$ interference filters from OCLI was used to check the dispersion properties of the modules. Because the filters transmitted far more light than the monochromator, they were used to search for ghost images. 


\section{IRS GROUND-BASED PERFORMANCE TESTING}

The methods used to verify the performance of the IRS prior to delivery and launch are described below.

\subsection{Detector properties}

The three fundamental detector properties, read-noise, dark-current and responsivity were measured with the modules in the flight condition in the Janis Dewar using the CE, S/C simulator, and TTACS. The dark- current and read-noise were measured by closing the LHe temperature door and making exposures at all available exposure times up to and including 500 seconds. The measured combined noise was below the requirement. The responsivity was measured in an end-toend way by exposing the modules to the PBB at a temperature of $60^{\circ} \mathrm{C}$ and then blocking the PBB and making a second exposure to the room radiation at a temperature of $23^{\circ} \mathrm{C}$. Extracting the responsivity from these measurements required knowledge of the transmission of the ND filters in the Dewar. The transmission curves were measured by the vendors, Janos and DSI, and they were independently measured at the University of Rochester by Dan Watson. The total ND stack varied from $\mathrm{ND}=4$ for the S-H to ND $=6$ for the S-L peak-up. The end-to-end transmission agreed with model calculations.

Each module contains a flood illuminator to monitor the responsivity stability ${ }^{2}$. Data taken over several months show the response to the stimulator remained constant $\leq 1 \%$.

\subsection{Spectral resolution}

The module spectral resolution was measured by the monochromator, and by measuring the line widths from gas cell absorption spectra. $\mathrm{H}_{2} \mathrm{O}, \mathrm{CO}_{2}$ and $\mathrm{NH}_{3}$ were used as absorbers. The $\mathrm{H}_{2} \mathrm{O}$ spectrum was obtained by subtracting pairs of exposures taken with the PBB-to-Dewar distances differing by $300 \mathrm{~mm}$. The slight difference in the atmospheric absorption over this $300 \mathrm{~mm}$ path was sufficient to achieve good signal to noise. The $\mathrm{CO}_{2}$ and $\mathrm{NH}_{3}$ spectra were obtained by placing a block of dry ice or a dish of household ammonia near the Janis Dewar window. A small cardboard shield prevented room air currents from dispersing the gasses too quickly. The resolutions listed in Table 1 were achieved.

\subsection{Wavelength coverage}

The data from the spectral resolution tests were also used to derive dispersion curves for the low-resolution modules. Similar data for the high-resolution modules were compared to the Code V raytraces for the modules. In all cases the observed and predicted wavelength ranges agreed. The only deviation from the formal requirements was that the short wavelength limit of the S-L module was 5.2 microns rather that the specified 5 microns. This deviation was driven by the need to avoid out-of-order leakage in the S-L.

\subsection{Sensitivity}

Verification of the photometric sensitivity was accomplished by measuring the differential response to the PBB and the ambient $\mathrm{BB}$ radiation from the room.

\subsection{Focus}

The focus verification entails two different aspects of the IRS: the IRS focus position relative to the CTA focus, and the focus within the individual modules. The first is determined warm. Because all the modules have entrance slits, or apertures in the case of the peak-up, preceding the blocking filters in their optical paths, the slits and apertures can be directly viewed from outside using an optical microscope. Because the system is all aluminum, the CTE mismatch is very small, and thermal induced strains are much smaller than the CTA depth of field.

The focus within the modules was verified with the modules cold. This was accomplished by observing the sharpness of narrow spectral features in the gas absorption spectra, by observing the sharpness of the edges of the peak-up windows and the ends of the slits in detector images. In the first case the Code V PSF could be used to compare the sharpness of 
the spectral feature for a point source illumination. In the second case, the Laplace transform of the Code V PSF was compared to the observed edge drop offs.

\section{6 f-beam mapping}

The PBB was used to map the shape and size of the f-beam. With the PBB at the nominal position of the CTA exit pupil relative to the entrance apertures, it was scanned in $\mathrm{X}$ and $\mathrm{Y}$ (see Figure 4) to find the maximum response for each module. Once the maximum was found, scans were made to map the shape of the f-beam. These scans were compared to model plots of the anticipated f-beam at the CTA exit pupil. Consistent agreement was achieved.

\subsection{Scattered/stray light}

Because the IRS spectrographs do not contain Lyot stops, they have significant sensitivity to radiation outside their $\mathrm{f} / 12$ acceptance cones. The out-of-the-f-cone response was measured with the PBB by scanning it in X, Y, and Z. The system, IRS plus CTA, off-axis response was calculated by convolving the measures out-of-the-f-cone response with the predicted stray light distribution within the CTA.

\section{IN-FLIGHT OPERATION AND CALIBRATION}

Uncertainties in the transmission of the neutral-density filter stacks prevented a useful determination of the overall sensitivities prior to launch. Therefore, the sensitivities of the modules were predicted by analytic models. Extensive observations using a prototype of the Short-High module at the Hale $5 \mathrm{~m}$ telescope verified the procedures used in the sensitivity predictions to the $20 \%$ level $^{7 \& 8}$.

Mapping the relative positions of each field of view and the spacecraft's Pointing Calibration Reference Sensor (PCRS) was the most critical step of the In-orbit Checkout (IOC) phase ${ }^{5}$. The positions must be measured to an accuracy of better than 0.14 arcsec radial ( 0.28 for the long-wavelength modules) to meet the $5 \%$ radiometric requirements. The positions were measured iteratively, starting with ground-based estimates and proceeding successively through ultracoarse, coarse, and fine focal-plane surveys. Combined with the determination of the focus between the telescope and the slits, this process took place over nearly six of the eight weeks of the IOC period. The estimated uncertainties in the final measured positions of the slits and the peak-up arrays are better than the requirements in all cases, ranging from 0.09 to 0.12 arcsec.

Our knowledge of the internal focus and optical calibration of the spectral orders were updated in-flight using a combination of photometric standard stars and emission line objects. The widths of the orders were derived from the zodiacal light at maximum intensity, while order curvatures, tilts, and wavelength solutions were re-derived from spectral maps of emission line stars such as P Cygni and planetary nebulae such as NGC 6543, NGC 7027, and SMP 083. Figure 5 presents the hydrogen recombination spectrum of the Be star $\gamma$ Cas, which provides a good check of the wavelength calibration for Short-High.

Flat-fielding and spectrophotometric calibration are determined from mapping and staring observations of well known standards, including $\xi$ Dra (HR 6688, K2 III), HR 7310 (G9 III) and HR 6606 (G9 III). Calibration of the low-resolution modules also used fainter standards (e.g. HD 42525, A0 V). Morris et al. provide more details about the calibration scheme $^{9}$. Decin et al. discuss the stars and synthetic spectra used for in-flight spectrophotometric calibration ${ }^{10}$. We are also verifying the calibration using spectral templates generated as described by Cohen et al. and observations of additional standard stars ${ }^{11}$.

The overlaps in wavelength coverage between the various modules and in orders within each module aid the internal cross-calibration of the IRS. These overlaps were also used to search for leaks in the order-sorting filters by observing "cold" and "hot" sources in two overlapping orders. In the case of the first-order filter for Long-Low (LL1), which had partially delaminated prior to launch, we used the combination of Uranus and Neptune as the cold sources and spectrophotometric standard stars as the hot sources. At this time the analysis limits any possible filter leak in LL1 to a maximum of $\sim 5 \%$ for a Rayleigh-Jeans spectrum. Further on-going efforts will refine this limit. 
The third largest solar proton flare in the past 25 years began on 2003 October 28, resulting in an integrated proton flux through the arrays of $1.9 \times 10^{9}$ protons $\mathrm{cm}^{-2}$ to the 50 percentile dose expected during the first 2.6 years of the mission. As a result, the sensitivity of $\sim 4 \%$ of the pixels in Long-High and $\sim 1 \%$ of the pixels in the other modules have been degraded. The degree of damage is consistent with the pre-launch damage experienced by non-flight arrays that were exposed to a beam of $40 \mathrm{MeV}$ protons at the Harvard Cyclotron Facility. The IRS is more sensitive to damage than the other science instruments on board Spitzer because it is operating at lower background conditions where even a small increase in dark-current has a significant effect.

The measured responsivity of the system in flight was on average two times better than the pre-launch model prediction. The detector noise measured in the unilluminated parts of the arrays are the same as measured pre-launch. Therefore, potentially in the limit of small signals the sensitivity is on average two times better than the model predictions. Most of this increase is due to design margin, an assumed factor to account of errors and non-modeled effects. The assumed margins were a factor of root two for the short wavelength modules and a factor of two for the long wavelength modules.

Updated estimates of sensitivity are available in the current version of the $\mathrm{SOM}^{5}$. The SSC provides a sensitivity calculator for the IRS known as SPEC-PET that includes detector noise, source shot noise, and background shot noise ${ }^{3}$. At the current time the sensitivity is limited by systematic effects, such as noise from the flat-field, fringing, and/or extraction edge effects, which are not included in the calculations of SPEC-PET. The observed sensitivities are approximately three times worse than would be achieved by the above responsivities and detector noise. It is anticipated that the realized sensitivities will improve as the system calibration improves.

\section{USING THE IRS}

The IRS has two spectroscopy and one imaging modes. In addition, there is a mode to image a target or an offset star and then move the target to the designated slit(s). These modes are described below.

\subsection{Spectroscopy}

The IRS currently has two modes of spectroscopic operation, the spectral "Staring" and "Mapping" modes. In both cases the target is acquired and then observed in a fixed sequence starting with SL2 (Short- Low second-order), SL1, SH, LL2, LL1, LH, skipping any un-requested slits.

The target acquisition method to be used is selected by the observer. One may simply rely on the blind telescope pointing to point the slits to the specified position on the sky. A second option is to use the PCRS to calculate the centroid of a reference star, use the offset between the star and the science target and move the science target into the $\mathrm{slit}^{12}$. A third and more accurate option is to use the IRS peak-up cameras to centroid on a 16 or $22 \mu \mathrm{m}$ image of the science target or a reference star before commencing the requested spectroscopic observations (see §7.2). Using the PCRS or IRS peak-up on a reference star requires accurate coordinates for both the offset star and the science target.

The IRS staring is the more basic mode of operation, and it results in observing the target at the $1 / 3$ and $2 / 3$ positions along the slit (its two nod positions), each with the integration time specified for that slit. The mapping mode steps the slit parallel and/or perpendicular to the slit according to the number and the size of the steps specified by the observer for each slit. The mapping mode does not perform the $1 / 3$ and $2 / 3$ nod observations that are automatically done in the staring mode.

Both the staring and mapping modes accept multiple target/position inputs, as long as all of the positions are within a region two-degree radius in the sky. Multiple targets are specified in a "cluster" list by either their 1) absolute positions, 2) right ascension and declination offsets or 3) parallel/perpendicular slit offsets with respect to an absolute fiducial position. If using IRS in spectral mapping mode with cluster inputs, parallel/perpendicular slit offsets cannot be used. If the cluster specification is selected, all sources/offsets in the cluster are observed with the same slit before proceeding to the next slit. Use of the cluster specification, as opposed to repeating the same observation one source at a time, reduces the observatory overhead and can lead to substantial savings in total "wall clock" time. 
The SOM describes the IRS observing modes and the various methods of target acquisition in more detail. In the sections below, we concentrate on using the IRS peak-up cameras to place a target in the desired slit, and describe how an observer can use the peak-up fields for imaging at 16 and $22 \mu \mathrm{m}$.

\subsection{IRS peak-up}

The IRS SL module contains two peak-up imaging fields. Their field of view is $\sim 55 \times 80 \mathrm{arcsec}$ with a scale of $1.8 \mathrm{arcsec}$ per pixel, and their bandpasses are centered at $16 \mu \mathrm{m}$ and $22 \mu \mathrm{m}$ for the "blue" and "red" camera respectively (see Table 1). The IRS peak-up mode enables the placement of a source on a spectrographic slit or series of slits more accurately than just using blind pointing of the spacecraft alone. The telescope blind pointing has a positional accuracy of 1 arcsec. ( $1 \sigma$ RMS, radial). An on-board algorithm determines the centroid of the brightest source in the specified peak-up field and communicates the offsets required to accurately position the target in the requested slit to the spacecraft. As long as the coordinates of a target are accurate enough to place it on the peak-up imaging field and it is the brightest object in the field, the IRS will accurately offset to the selected slits. The peak-up images are supplied to the observers and can also be used scientifically if desired.

The allowed ranges of flux densities for blue and red peak-up point sources are $\mathrm{f}$-blue $=0.8-150 \mathrm{mJy}$ and f_red $=1.4$ $340 \mathrm{mJy}$, respectively. However, to avoid excessive integration times and a higher probability of failure, we recommend a flux of at least $2 \mathrm{mJy}$ for the blue and $5 \mathrm{mJy}$ (The SSC is now recommending in their memos that the observer only peak up on objects greater than $10 \mathrm{mJy}$ for both PU arrays.) for the red. The IRS peak-up algorithm has been optimized for point sources (and indeed this mode has been the most extensively tested and verified in-orbit), but an "Extended Source" mode is available

The observer can specify two peak-up accuracies: "High" and "Medium". In the simplest case, when peaking up on the

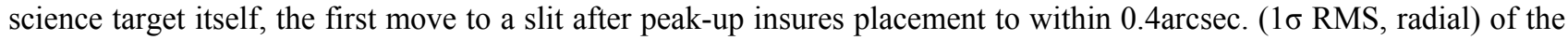
slit center for High Accuracy and 1.0arcsec. for Medium Accuracy. The High Accuracy value is driven by the 5\% radiometric accuracy requirement for the Short-High slit (4.7 arcsec. width). The IRS section of the SOM provides details on how these accuracy options apply to multiple slit positioning after peak-up (e.g., for targets in IRS cluster mode).

In the event that the science target cannot also serve as the peak-up target (e.g. if its flux is too low or too high), the "Offset Peak-up" mode gives the option of peaking-up using a nearby source. The observer is free to use any suitable source within 30arcmin of the science target, but the Spitzer planning tool (SPOT) also provides a list of recommended candidates ${ }^{13}$. Note that this mode requires accurate coordinates, including proper motions, for both the offset and science target. An IRS peak-up returns the image of the peak-up target.

The PCRS extends the IRS peak-up capability to optical point sources with visual magnitudes ranging between $\mathrm{m}_{\mathrm{V}}=7$ and 10. The only mode for PCRS currently available provides pointing equivalent to the High Accuracy mode of the IRS. The PCRS peak-up does not produce or return an image.

\subsection{Imaging with the IRS peak-up arrays}

The peak-up fields in the Short-Low module can provide a means of obtaining images at 16 and $22 \mu \mathrm{m}(5.4$ and $7.5 \mu \mathrm{m}$ FWHM respectively). The $16 \mu \mathrm{m}$ window in particular is interesting because the other cameras on Spitzer do not cover this wavelength region, and because this wavelength region was used by the Infrared Space Observatory (ISO) for most of its deep extragalactic surveys. Since these surveys probed the properties of galactic evolution only up to $z \sim 1$, IRS can provide the link between these past results and the new discoveries which will be made by Spitzer at $z \sim 2$.

Imaging with the IRS peak-up cameras has not been fully supported for the first year of operations, but the SSC will provide the ability to obtain large mosaics in a manner similar to IRAC before the end of $2005^{14}$. However, an interim method called CHEAP (Cornell High-Efficiency Advanced Peak-up) has been used extensively to obtain mid-infrared images. CHEAP is based on an IRS Short-Low staring observation and uses our knowledge of the offsets between the center of the peak-up windows and the Short-Low slits in spacecraft coordinates, along with the standard two-position nodding along the slit, to place a source target in various positions on the two peak-up windows. The user may select any 
combination of exposure times and cycles of the Short-Low to obtain an image. The CHEAP data are processed by the standard SSC pipeline as if they were standard Short-Low spectroscopic observations. The software that combines and calibrates the produced images is already available.

As an example, taking a CHEAP image using a standard $60 \mathrm{sec}$ Short-Low staring exposure will produce two images at 16 and $22 \mu \mathrm{m}$ with an RMS noise of $30 \mu \mathrm{Jy}$ in only $720 \mathrm{sec}$ of total time. It is interesting to note that the RMS noise of the ISO observations of the Hubble Deep Field (HDF) was just $\sim 13 \mu \mathrm{Jy}$ at $15 \mu \mathrm{m}$ and that the faintest source detected in the HDF at $15 \mu \mathrm{m}$ was $\sim 50 \%$ brighter than the sensitivity in the above example ${ }^{15}$. The use of CHEAP to obtain 16 and $22 \mu \mathrm{m}$ images of a sample of high redshift $(z \sim 1.5)$ submillimeter galaxies is presented by Charmandaris et al. ${ }^{16}$ and the first $16 \mu \mathrm{m}$ imaging of a Lyman Break Galaxy at $z=2.79$ is discussed by Teplitz et al. ${ }^{17}$.

\subsection{Planning IRS observations}

A number of issues should be considered in the design of spectroscopic observations using IRS. These are addressed in detail in the SOM, but we briefly mention a few of the more important ones in this section.

Although the infrared background is fainter than the ground based background by a factor of $10^{6}$, it may still be necessary to take an "off source" measurement of the background. The low-resolution modules do this automatically in the sense that both sub-slits are exposed at the same time so the spectrum from the "off" slit can be subtracted from the "on" slit to remove the background. Alternately, the low-resolution slits are long enough to do the background subtraction on the slit itself. However, the high-resolution slits are too short to subtract the background by differencing the nod positions. If the continuum level is important, off source integrations using the same module are required for the purpose of background subtraction. SPOT provides an estimate of the background that is helpful in planning for background subtraction. As a general rule, the observers should use the low-resolution modules to measure continuum and broadband features, and the high-resolution modules to measure unresolved lines.

The peak-up system works extremely well over its range of parameters. However, the observer needs to carefully check that the flux of the peak-up candidate falls within the specified limits of the cameras and correctly enter it into SPOT. Furthermore, as already mentioned in section 7.2 the candidate must be the brightest object within $\sim 120$ arcsec to ensure that it will be the one selected by the peak-up algorithm. During the early phase of the Spitzer mission there have been several peak-up failures due to violations of one or the other of the above requirements.

Observing with Spitzer is unlike most ground based infrared observing. On the ground the background signal overwhelms the source by many orders of magnitude. Therefore, the system noise is set by the shot noise from the background. On Spitzer, the noise (N) is dominated by detector noise for small signals (S), so $\mathrm{N} \sim$ constant. However, as the signal level increases the shot noise in the signal eventually dominates: $N \sim S^{1 / 2}$. At the highest signal levels fixed pattern noise (flat-fielding errors, etc.) are dominant and consequently $\mathrm{N} \sim \mathrm{S}$. At the present time it appears that the flatfielding term is 1 to $2 \%$, effectively limiting the maximum $\mathrm{S} / \mathrm{N}$ ratio to $\sim 50$ to 100 . Similarly at very low signals the dark-field uncertainty limits our $1 \sigma$ detection to $\sim 0.3 \mathrm{mJy}$ at $16 \mu \mathrm{m}$ for low backgrounds ${ }^{18}$. The online $\mathrm{S} / \mathrm{N}$ estimator includes all but this last term.

\section{PIPELINE DATA PROCESSING}

As described in detail in the SOM, IRS observations, either in DCS or Raw mode, are stored as FITS files of what is called a Data Collection Event (DCE). A DCE contains all the data obtained by an IRS module since the most recent destructive read. The IRS Science Pipeline at the SSC treats each DCE independently. The processing of DCS data, such as those collected during an IRS peak-up, by the pipeline is minimal and all processing is performed on-board the spacecraft. However, for data obtained in Raw mode the pipeline removes basic instrumental signatures and corrects for variations in spectral response within and between spectral orders. A number of these steps, such as corrections/checks for saturation, cosmic rays, dark- current subtraction as well as linearization are performed in the cube level prior to fitting a slope to the sampled up the detector charge integration ramp. Others, such as correction for drifts in the darkcurrent, and stray light or crosstalk between the orders are applied once the slope image has been created. 
The IRS array data supplied in FITS files to the observer are organized into four categories: Engineering Pipeline Data, Basic Calibrated Data (BCD), Browse-Quality Data (BQD), and Calibration Data. Additional files are also available of the masks used in the pipeline processing as well as processing log and quality assurance files. The Engineering Pipeline data consist of the raw detector sample images, with some descriptive information in the FITS file headers. The BCD files are the fundamental basis for science analysis, with the primary product being a two-dimensional slope image of each DCE in units of electrons $\sec ^{-1}$ pixel $^{-1}$, accompanied by a header containing the essential programmatic information and the processing, calibration, and pointing history. Additional BCD data files include uncertainty images that accompany each slope image and various intermediate images that do not have all pipeline processing corrections applied. The BCDs also include coadded image data for those observations with more than one ramp at a sky location. It is expected that the BCD files will be the standard data input for subsequent publishable science data analysis. As an example, in a standard IRS staring observation in which the observer has requested 3 cycles of $30 \mathrm{sec}$ integrations on a science target with the SH module will result in 6 DCEs in Raw mode. The Engineering Data pipeline will process the data, which will have the form of six data cubes ( 3 integration cycles at each of the two nod positions along the slit), each $128 \times 128$ pixels x 16 detector reads in size.

The BCD pipeline will produce six 128x128 BCD slope images as well as two $128 \times 128$ co-added slope images. The Calibration Data include measurements of standard calibration objects and can be used with the BCD to further refine the quality of the science data. The BQD is designed to provide the observer with an early look at spectra extracted from 2-dimensional BCD images of point sources. In general, the BQD spectra are designed to convey the richness of the observed data and in some cases may be of publishable quality. To extract spectra from each individual BCD image the post-BCD pipeline first applies a search algorithm to trace the spectrum in the two dimensional 128x128 pixel images. Extraction is performed using an aperture that follows the linear expansion of the point-spread function (PSF) with wavelength. All spectra are extracted as point sources and no sky background is subtracted before the extraction is made (see discussion in $§ 7.1$ on how this may affect the extracted spectrum). The spectra are then photometrically calibrated (in Janskys) to remove the effects of truncation of the PSF by the slit aperture and high-order systematic residuals from flat-fielding.

Most pipeline operations are now routine, but the need to characterize the performance of the instrument in-orbit has led to numerous modifications to the algorithms and adjustments to parameters. Most important are ongoing efforts to refine the detection and mitigation of latencies and cosmic ray hits, correct for stray light between the echelle orders, and remove stray light in Short-Low module originating from the peak-up apertures. Extraction methods and the final spectrophotometric calibration of the spectra are also very sensitive to the pixelation of PSF at each wavelength, and as a result both the extraction and calibration will require some fine-tuning as a result of what has been learned from in-flight measurements. A detailed user's guide to the SSC pipeline and its products is in preparation.

\section{SPECTROSCOPY MODELING, ANALYSIS AND REDUCTION TOOL}

The IRS Spectroscopy Modeling, Analysis and Reduction Tool (SMART) is an IDL software package used to reduce and analyze IRS data from the four modules and the two peak-up arrays ${ }^{19}$. SMART is designed to operate on the basic calibration data (BCD) delivered by the SSC pipeline and can be run both interactively and in batch mode. It provides a suite of quick-look routines that enable observers to assess the quality of their data.

SMART extracts spectra from either individual or coadded BCDs, and these can be weighted by a pixel- mask that characterizes the noise in the data. The extraction routines include column extraction of a point source scaled to the instrumental PSF, fixed-aperture extraction for extended sources and a weighted Gaussian extraction for point sources.

Several options are available to optimize the sky subtraction. The spectra from the individual modules can be combined to produce a single spectrum from 5-38 $\mu \mathrm{m}$. Spectral analysis, including line, black-body, template and zodiacal light fitting, photometry, defringing and dereddening are also available. The spectral analysis package is based on the ISO Spectral Analysis Package, ISAP ${ }^{20}$. SMART includes packages developed by the Spitzer Legacy teams. The C2D team provided a number of defringing tools while the FEPS team has contributed IDP3, an image analysis software package originally developed for the NICMOS instrument of the Hubble Space Telescope.

Higdon et al. provide more details about the SMART package ${ }^{19}$. It will become publicly available in September 2004. 


\section{SAMPLE RESULTS}

The articles in the special issue of the Astrophysical Journal Supplement Series devoted to the first Spitzer results* provide many examples of the quality of the spectra from the IRS. In addition to the spectrum of $\gamma$ Cas in Figure 5, we show examples of one low-resolution spectrum and one high-resolution spectrum, both of galaxies. All data presented have been processed and extracted from individual DCEs using the IRS pipeline at the SSC. Figure 6 presents a spectrum of NGC 7714 using Short-High and Long-High (240 seconds integration each). One can immediately note the many forbidden lines and dust features present ${ }^{21}$. Figure 7 presents the spectrum of UGC 5101 obtained using the low-resolution modules. The integration times were 12 seconds in each Short- Low sub-slit and 28 seconds in each Long-Low sub-slit, and this was sufficient to show a rich spectrum with gas, dust, and ice features ${ }^{22}$.

\section{CONCLUSIONS}

The IRS is a major step forward in speed and sensitivity. It clearly enables the extension of infrared spectroscopy to a very large number of extragalactic sources. The designed spectral resolution is well matched to the expected line widths from these objects. The IRS also allows mid-infrared spectroscopy of objects such as brown dwarfs, individual stars in neighboring galaxies, and a wide variety of other sources previously difficult or impossible to study spectroscopically in the mid-infrared. This description of the IRS gives the potential observer a brief review of its design, its capabilities, and how to use it. Now it is up to the astronomical community to fully exploit what the IRS can do.

*Nearly one hundred papers describing the facility, its instruments, and the early science results appear in the Sept. 1, 2004 ApJS Volume 154.

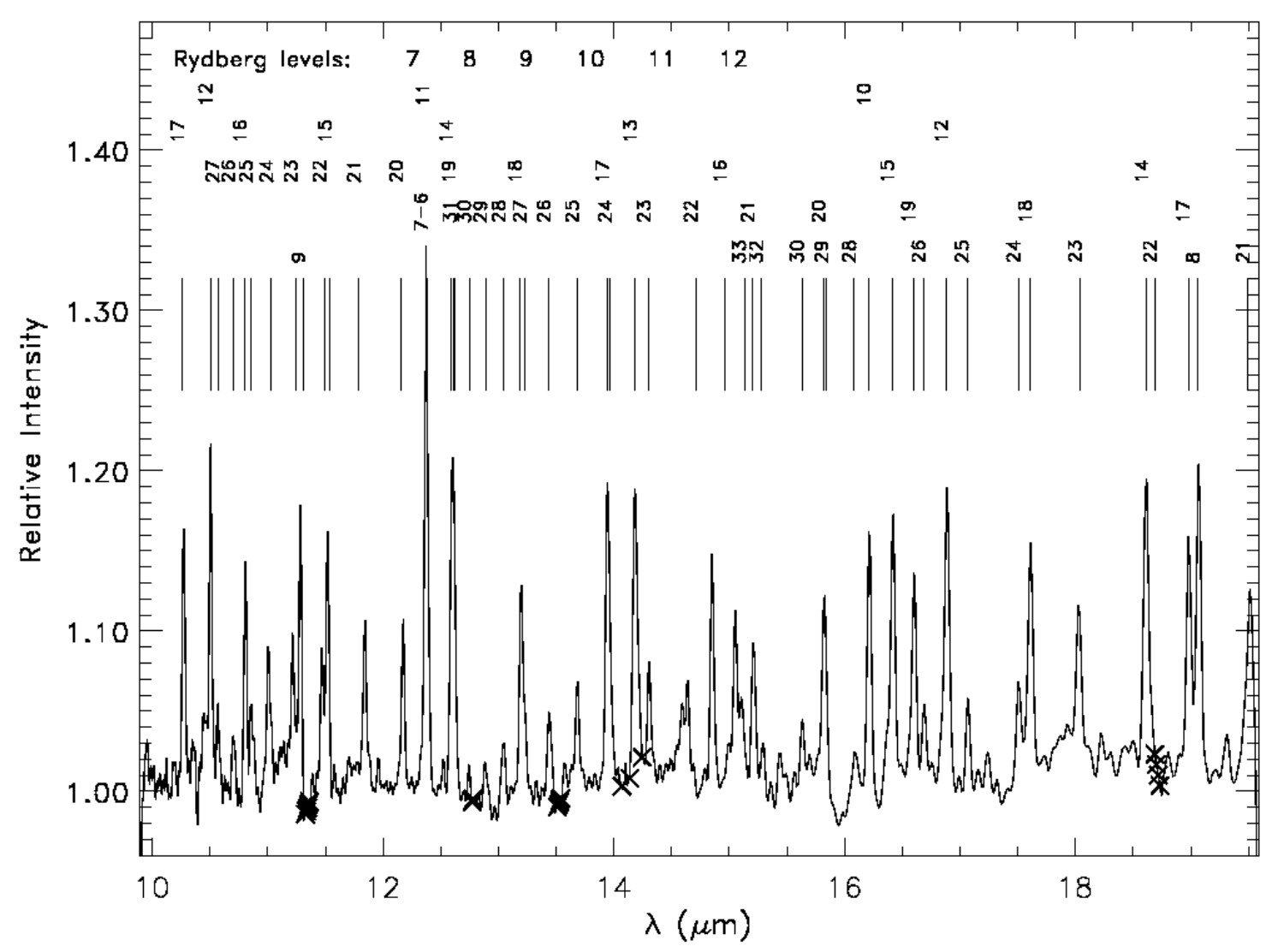

Figure: 5 The short high spectrum of the emission line star $\gamma$ Cass. This object was used to do the wavelength calibration of the short high module. The baseline has been removed. 


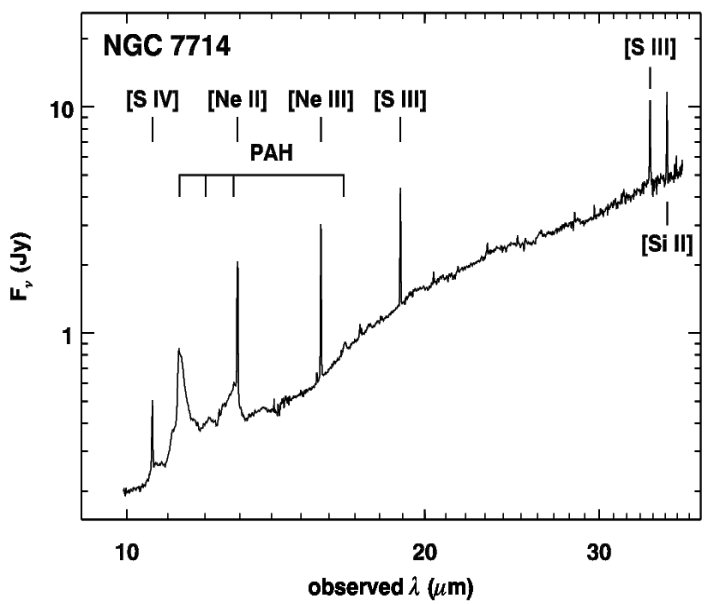

Figure 6: The high-resolution spectrum of the starburst galaxy, NGC 7714

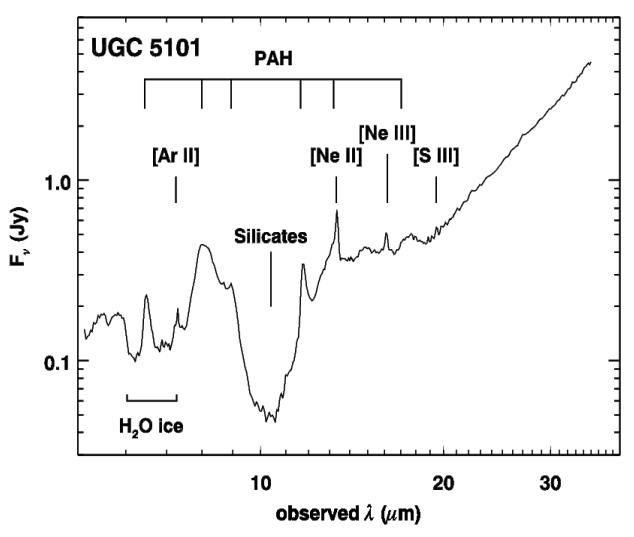

Figure 7: The complete low resolution, short low and long low spectra, of the Ultra Luminous Infrared Galaxy (ULIRG) UGC 5101.

\section{ACKNOWLEDGEMENTS}

The design, fabrication, and testing of the IRS at Ball Aerospace Corporation was supported by a NASA contract awarded by the Jet Propulsion Laboratory to Cornell University (JPL contract number 960803). The IRS pipeline was developed at the SSC at the California Institute of Technology under contract to JPL.

\section{REFERENCES}

1. J.R. Houck, T.L. Roellig, J. van Cleve, B.R. Brandl, \& K.I. Uchida, "IRS: the Spectrograph on SIRTF; Its Fabrication and Testing," 2000, SPIE, 4131, 70.

2. T.L. Roellig, et al., "Infrared Spectrograph for the Space Infrared Telescope Facility (SIRTF)," SPIE, 1998, $3354,1192 \mathrm{R}$.

3. J.R. Houck, \& J.E. van Cleve, “IRS: an Infrared Spectrograph for SIRTF,” SPIE, 1995, 2475, 456H.

4. R.J. Brown, J.R. Houck, \& J.E. van Cleve, "Optical Design for a High-Resolution Spectrometer for SIRTF/IRS," SPIE, 1996, 2863, 222B.

5. Spitzer Science Center, "Spitzer Space Telescope Observer's Manual-Version 4.0," (SOM) Pasadena, CA. http://ssc.spitzer.caltech.edu/documents/SOM/.

6. J.E. van Cleve, T.L. Herter, R. Butturini, G.E. Gull, J.R. Houck, B. Purger \& J. Schoenwald, "Evaluation of Si:As and Si:Sb Blocked-impurity-band Detectors for SIRTF and WIRE," SPIE, 1995, 2553, 502V.

7. J. van Cleve, et al. "SCORE: A Mid-Infrared Echelle Format Spectrograph with No Moving Parts," PASP, 1998, $110,1479$. 
8. J.D.T. Smith, \& J.R. Houck, “A Mid-Infrared Spectral Survey of Galactic Wolf-Rayet Stars,” AJ, 2001, 121, 2115.

9. P.W. Morris, V. Charmandaris, T. Herter, L. Armus, J.R. Houck, \& G.C. Sloan, "The Calibration Legacy of the ISO Mission,” 2003, ESA SP-481, 113.

10. L. Decin, P.W. Morris, P.N. Appleton, V. Charmandaris, \& L. Armus, "MARCS-Model Stellar Atmospheres, and Their Application to the Photometric Calibration of the Spitzer-IRS," 2004, ApJS, Accepted.

11. M. Cohen, T.G. Megeath, P.L. Hammersley, F. Martin-Luis, \& J. Stauffer, "Spectral Irradiance Calibration in the Infrared, XIII," 2003, AJ, 125, 2645.

12. M.W. Werner, et al. "The Spitzer Space Telescope Mission,” 2004, ApJS, Accepted.

13. SPOT: http://ssc.spitzer.caltech.edu/propkit/spot/.

14. G. Fazio, G., et al., "The Infrared Array Camera (IRAC) for the Spitzer Space Telescope" 2004, ApJS, Accepted.

15. H. Aussel, C.J. Cesarsky, D. Elbaz, \& J.L. Starck, "ISOCAM Observations of the Hubble Deep Field with the PRETI Method," 1999, A\&A, 342, 313.

16. V. Charmandaris, et al., "Imaging of High Redshift Sub-mm Galaxies at 16 and 22um with the Spitzer/IRS Peak-up Cameras: Revealing a population at $\mathrm{z}>2.5$," 2004, ApJS, Accepted.

17. H.I. Teplitz, et al., "Rest-frame MIR detection of an extremely luminous Lyman Break Galaxy with the Spitzer IRS,” 2004, ApJS, Accepted.

18. S.J.U. Higdon, et al. "First mid-infrared spectrum of a faint high-z galaxy: Observations of CFRS 14.1157 with the Infrared Spectrograph on The Spitzer Space Telescope," 2004, ApJS, Accepted.

19. S.J.U. Higdon, et al. "SMART: a data analysis package for the IRS," 2004, PASP, Submitted.

20. E. Sturm, et al. "The ISO Spectral Analysis Package ISAP," 1998, ASP Conf. Ser. 145: Astronomical Data Analysis Software and Systems VII, 7, 161.

21. B.R. Brandl, et al. "Spitzer-IRS Spectroscopy of the prototypical starburst galaxy NGC7714," 2004, ApJS, Accepted.

22. L. Armus, et al. "Observations of ULIRGs with Spitzer/IRS: Early Results on UGC 5101, Mrk 463e, and Mrk 1014," 2004, ApJS, Accepted.

23. Spectroscopy-Performance Estimation Tool (SPEC-PET), http://ssc.spitzer.caltech.edu/tools/specpet. 